Research Paper

\title{
Factors associated with improvement in symptoms and quality of life for first-line EGFR-tyrosine kinase inhibitor treatment in patients with EGFR-mutated non-small-cell lung cancer - A multicenter prospective SMILE study
}

Yu-Feng Wei ${ }^{1}, 2$, Wen-Tsung Huang ${ }^{3}$, Tu-Chen Liu ${ }^{4}$, Jiunn-Min Shieh ${ }^{5}$, Chih-Feng Chian ${ }^{6}$, Ming-Fang Wu Chih-Cheng Chang, ${ }^{8,9}$, Ching-Hsiung Lin ${ }^{10}$, Jen-Chung Ko1, Chia-Mo Lin ${ }^{12}$, Te-Chun Hsia ${ }^{13,14 \bowtie}$

1. Division of Chest Department, Department of Internal Medicine, E-Da Hospital, I-Shou University, Kaohsiung, Taiwan.

2. Institute of Biotechnology and Chemical Engineering, I-Shou University, Kaohsiung, Taiwan

3. Division of Hematology-Oncology, Department of Internal Medicine, Chi Mei Medical Center Liouying, Tainan, Taiwan

4. Department of Chest Medicine, Cheng Ching Hospital-Chung Kang Branch, Taichung, Taiwan

5. Department of Internal Medicine, Chi Mei Medical Center - YongKang Branch, Tainan, Taiwan

6. Division of Pulmonary and Critical Care Medicine, Internal Medicine Department, Tri-Service General Hospital, National Defense Medical Center, Taipei, Taiwan

7. Division of Medical Oncology, Department of Internal Medicine, Chung Shan Medical University Hospital, and School of Medicine, Chung Shan Medical University, Taichung, Taiwan

8. Division of Pulmonary Medicine, Department of Internal Medicine, Shuang Ho Hospital, Taipei, Taiwan

9. Department of Internal Medicine, School of Medicine, College of Medicine, Taipei Medical University.

10. Division of Chest Medicine, Department of Internal Medicine, Changhua Christian Hospital, Changhua, Taiwan

11. Department of Internal Medicine, National Taiwan University Hospital - Hsinchu Branch, Hsinchu, Taiwan

12. Sleep Center, Pulmonary and Critical Care Medicine, Shin Kong Wu Ho-Su Memorial Hospital, Taipei, Taiwan

13. Department of Respiratory Therapy, China Medical University, Taichung, Taiwan

14. Division of Pulmonary and Critical Care Medicine, Department of Internal Medicine, China Medical University Hospital, China Medical University, Taichung, Taiwan

$\square$ Corresponding author: Te-Chun Hsia, MD, Telephone: +886-4-2205-2121 ext 1250, Fax: +886-4-2203-8883, E-mail: derrick.hsia@msa.hinet.net, Postal Address: Division of Pulmonary and Critical Care Medicine, Department of Internal Medicine, China Medical University Hospital, China Medical University, Yude Road, North District, Taichung City 40447, Taiwan

(c) The author(s). This is an open access article distributed under the terms of the Creative Commons Attribution License (https://creativecommons.org/licenses/by/4.0/). See http://ivyspring.com/terms for full terms and conditions.

Received: 2018.10.08; Accepted: 2019.04.28; Published: 2019.07.10

\begin{abstract}
Introduction: Epidermal growth factor receptor-tyrosine kinase inhibitors (EGFR-TKIs) are a standard first-line treatment for advanced EGFR-mutated NSCLC patients. Factors associated with symptoms and quality of life (QOL) improvements have not been investigated.

Methods: We conducted a multicenter, prospective study to evaluate improvements in QOL and symptoms in NSCLC patients treated with first-line EGFR-TKIs. QOL was assessed using the instrument of Functional Assessment of Cancer Therapy-Lung questionnaire (FACT-L) and Treatment Outcome Index (TOI). Assessment of symptoms was evaluated using the Lung cancer subscale (LCS).

Results: Eligible subjects included 280 patients for endpoint analyses. The mean FACT-L score increased by 4.0 \pm 15.56 at Week $2(p<0.001), 5.1 \pm 18.48$ at Week $4(p<0.001)$, and $4.2 \pm 20.27$ at Week $12(p=0.001)$. Similarly, a $2.3 \pm 11.65$ ( $p<0.001), 3.2 \pm 13.59(p<0.001)$, and $2.4 \pm 14.34(p=0.009)$ increase in mean TOI score were observed at Weeks 2,4 and 12, respectively. For LCS, it was slightly increased by $1.7 \pm 4.61,2.0 \pm 5.50$, and $2.0 \pm 5.36$ at Weeks 2,4 , and 12 (all $p<0.001$ ), respectively. Subgroup analyses showed patients who were ex-smokers or with at least 3 metastatic sites were associated with symptoms improvement. Patients who were ex-smokers, with at least 3 metastatic sites, a PS of 1 , or treated with gefitinib were associated with QOL improvement.
\end{abstract}

Conclusions: In EGFR -mutated NSCLC patients who were treated with first-line EGFR-TKIs, these ex-smokers or with 3 or more metastatic sites were associated with improvements in symptoms and QOL.

Key words: epidermal growth factor receptor tyrosine kinase inhibitor, non-small cell lung cancer, quality of life 


\section{Introduction}

Lung cancer is the leading cause of cancer deaths around the world, including Taiwan.[1] Non-small cell lung cancer (NSCLC) accounts for more than 85\% of all lung cancer cases and more than $70 \%$ of lung cancer patients present with advanced disease (stage III or IV) at initial diagnosis.[2]

NSCLC patients with EGFR mutation(s) have been observed to have a higher response rate, longer progression-free survival and better tolerability to EGFR-tyrosine kinase inhibitors (EGFR-TKIs) compared with standard first-line platinum-based doublet chemotherapy.[3-5] In addition to prolonged progression-free survival, controlling the symptoms and improving the quality of life (QOL) of patients are also key goals in the treatment of advanced NSCLC. Post-hoc analyses of large randomized trials demonstrated EGFR-TKIs, including gefitinib, erlotinib, and afatinib, were associated with better symptom control and QOL outcomes in EGFR -mutated NSCLC patients when compared to conventional chemotherapy.[6-9] In the LUX-Lung 7 trial, similar improvements in patient-reported outcomes and QOL were reported in patients treated with afatinib and gefitinib.[10] To the best of our knowledge, however, factors associated with improvements in symptoms and QOL with EGFR-TKI treatment have not been investigated. The aim of this study was to examine the extent patient characteristics have on the improvement of symptoms and QOL in a real-world patient population in Taiwan treated with EGFR-TKIs.

\section{Materials and Methods}

\section{Patient selection criteria and study design}

An open-label, non-interventional, single-arm, multicenter prospective observational study was conducted across 7 medical centers and 5 regional hospitals in Taiwan. Patients who fulfilled all of the following criteria were eligible for the study: 1) provision of informed consent form, 2) aged 20 years and older, 3) diagnosed with locally advanced or metastatic (stage IIIb/IV) NSCLC and confirmed positive for EGFR mutation, 4) treatment-naïve and ready for the prescription of a EGFR-TKI as their first-line cancer treatment, 5) able to complete the questionnaires. Patients were excluded from the study if they were involved in the planning and/or the progress of the clinical trials. The study was reviewed and approved by all the Institutional Review Board of the participating institutes.

EGFR-TKI therapy of $250 \mathrm{mg}$ gefitinib (Iressa ${ }^{\circledR}$, AstraZeneca, Cambridge, England), 150 mg erlotinib (Tarceva ${ }^{\circledR}$, Hoffmann-La Roche, Basel Switzerland) or
40 mg afatinib (Giotrif ${ }^{\circledR}$, Boehringer Ingelheim, Ingelheim, Germany) was prescribed to patients by physicians at baseline according to physicians' judgment under the real-world settings. Drugs were administrated daily by investigators and no patients changed medications during the course of the study.

\section{Data collection and measurements for symptoms and QOL}

Demographic and clinical data related to lung cancer were collected, including age, gender, smoking status (number and duration), staging at diagnosis, metastatic site, subtype of EGFR mutation, performance status (PS) and concomitant diseases at baseline. QOL was assessed using the Functional Assessment of Cancer Therapy-Lung (FACT-L) questionnaire and Treatment Outcome Index (TOI) derived from FACT-L. Improvement to QOL was defined as an increase in FACT-L or TOI score by $\geq 6$ points of change from baseline.[11] Improvement in disease related symptoms was measured by the Lung Cancer Subscales (LCS) of FACT-L questionnaire. A clinically meaningful improvement in symptoms was defined as an increase in LCS by $\geq 2$ points from baseline.[12] An LCS increase/decrease of $\leq 2$ points from baseline was defined as no change. Worsening in symptoms was defined as a decrease in LCS by $\geq 2$ points from baseline.[12] The instrument was completed at pre-treatment (baseline), every 2 weeks in the first month, and 3 months after administration of EGFR-TKIs.

\section{Statistical analysis}

Data are presented as mean \pm SD (or SE) for continuous parameters, and as number and percentage for categorical parameters. To evaluate improvement in symptoms, the evaluable-forsymptom improvement (EFS) population was used for data analysis, which was defined as patients with an evaluable baseline LCS assessment and at least one evaluable post-baseline assessment. The mean change in FACT-L, TOI, and LCS scores at Weeks 2, 4, and 12 from baseline was analyzed within the EFS population using the paired sample t-test. For subgroup analyses, the number and proportion of patients who achieved improvement during the study period was calculated by each subgroup with an exact 95\% confidence interval, to clarify the factors associated with the improvement in symptoms and QOL in patients treated with EGFR-TKI.

All tests for significance were two-sided, and a p-value of less than 0.05 was considered to be statistically significant. All analyses were performed using Statistical Analysis System ${ }^{\circledR}$ (SAS) for Windows (Version 9.3 or higher, SAS Institute, Cary, North Carolina, USA) statistical package. 
Table 1. Demographic information and baseline characteristics of patients.

\begin{tabular}{|c|c|c|}
\hline Characteristics & & EFS population, $N=280$ \\
\hline Age (year) & Mean \pm SD & $65.3 \pm 12.38$ \\
\hline \multirow[t]{2}{*}{ Gender } & Male & $103(36.8)$ \\
\hline & Female & $177(63.2)$ \\
\hline \multirow[t]{5}{*}{ Smoking Status } & Never Smoke & 207(73.9) \\
\hline & Ex-smoker & $42(15.0)$ \\
\hline & Current smoker & $27(9.6)$ \\
\hline & Occasionally smoke & $3(1.1)$ \\
\hline & Missing & $1(0.4)$ \\
\hline \multirow[t]{2}{*}{ Staging at enrollment } & IIIB & $26(9.3)$ \\
\hline & IV & $254(90.7)$ \\
\hline \multirow{7}{*}{$\begin{array}{l}\text { Metastatic sites at } \\
\text { enrollment }\end{array}$} & Bone & $106(37.9)$ \\
\hline & Lung & 102(36.4) \\
\hline & Brain & $58(20.7)$ \\
\hline & None & $28(10.0)$ \\
\hline & Liver & $23(8.2)$ \\
\hline & Adrenal & $6(2.1)$ \\
\hline & Other & $78(27.9)$ \\
\hline \multirow[t]{2}{*}{ EGFR mutation ${ }^{\#}$} & Common mutation* & $265(94.6)$ \\
\hline & Uncommon mutation & $15(5.4)$ \\
\hline \multirow[t]{3}{*}{ EGFR-TKI therapy } & Gefitinib & $202(72.1)$ \\
\hline & Erlotinib & $54(19.3)$ \\
\hline & Afatinib & $24(8.6)$ \\
\hline \multirow{4}{*}{$\begin{array}{l}\text { WHO performance } \\
\text { status }\end{array}$} & 0 & $125(44.6)$ \\
\hline & 1 & $110(39.3)$ \\
\hline & 2 & $34(12.1)$ \\
\hline & $3-4$ & $11(3.9)$ \\
\hline
\end{tabular}

Data were presented as N (\%)

*Common EGFR mutations are defined as mutations in exon 19 or 21; uncommon mutations are defined as mutations in exon 18 or exon 20

EFS $=$ evaluable-for-symptom improvement

Table 2. Changes in symptoms (LCS) and QOL (FACT-L and TOI) response of EFS population following EGFR-TKI therapy.

\begin{tabular}{|c|c|c|c|c|}
\hline \multicolumn{2}{|c|}{ Summary of response } & \multirow{2}{*}{$\begin{array}{l}\text { Week } 2 \\
280\end{array}$} & \multirow{2}{*}{$\begin{array}{l}\text { Week } 4 \\
270\end{array}$} & \multirow{2}{*}{$\begin{array}{l}\text { Week } 12 \\
251\end{array}$} \\
\hline $\begin{array}{l}\text { Changes from } \\
\text { baseline by } \\
\text { visits }\end{array}$ & $\begin{array}{l}\text { EFS } \\
\text { population }\end{array}$ & & & \\
\hline \multirow[t]{3}{*}{ Response $^{a}$} & Improvement & $128(45.7)$ & $122(43.6)$ & $125(44.6)$ \\
\hline & $\begin{array}{l}\text { Stable/No } \\
\text { change }\end{array}$ & $91(32.5)$ & $99(35.4)$ & $88(31.4)$ \\
\hline & Worsening & $61(21.8)$ & $59(21.1)$ & $67(23.9)$ \\
\hline \multirow[t]{2}{*}{ Changes in LCS } & Mean \pm SE & $1.7 \pm 0.28$ & $2.0 \pm 0.33$ & $2.0 \pm 0.34$ \\
\hline & $\mathrm{p}$-value & $<0.001$ & $<0.001$ & $<0.001$ \\
\hline \multirow{2}{*}{$\begin{array}{l}\text { Changes in } \\
\text { FACT-L }\end{array}$} & Mean \pm SE & $4.0 \pm 0.93$ & $5.1 \pm 1.12$ & $4.2 \pm 1.28$ \\
\hline & p-value & $<0.001$ & $<0.001$ & 0.001 \\
\hline \multirow{2}{*}{$\begin{array}{l}\text { Changes in } \\
\text { TOI }\end{array}$} & Mean \pm SE & $2.3 \pm 0.70$ & $3.2 \pm 0.82$ & $2.4 \pm 0.91$ \\
\hline & p-value & $<0.001$ & $<0.001$ & 0.009 \\
\hline
\end{tabular}

a Improvement is defined as an increase in LCS $\geq 2$ points; worsening is defined as a decrease in LCS $\geq 2$ points; stable/no change is defined as a change in LCS between

-2 and 2 points. Data were presented as N (\%)

$\mathrm{b}$ Change in TOI $\geq 6$ points from baseline score indicates a clinically relevant improvement to QOL.

c Change in FACT-L $\geq 6$ points from baseline score indicates a clinically relevant improvement to QOL.

EFS = evaluable-for-symptom improvement; LCS = Lung cancer subscale; FACT-L = Functional Assessment of Cancer Therapy-Lung questionnaire; TOI = Treatment Outcome Index

\section{Results}

Between November $7^{\text {th }} 2013$ and June 30th 2015, a total of 346 patients with NSCLC were screened for study entry. 292 patients $(84.4 \%, 292 / 346)$ met all screening criteria and were enrolled in this study. Of these patients, 280 were included in the EFS population for endpoint analyses. Demographic and characteristics of the EFS population are presented in Table 1. The average age was $65.3 \pm 12.38$ years old, ranging from 31.1 to 90.4 years. A large proportion of the EFS population was female $(63.2 \%)$ and there was a greater proportion of never-smokers $(73.9 \%)$ than former and current-smokers. Most patients had a PS score of $0-1(83.9 \%)$. The major subtypes of EGFR mutation were exon 21 L858R (50.3\%) and exon 19 $(44.3 \%)$ deletion. For treatment, $72.1 \%, 19.3 \%$ and $8.6 \%$ of patients were treated with gefitinib $250 \mathrm{mg}$ erlotinib $150 \mathrm{mg}$ and afatinib $40 \mathrm{mg}$, respectively. Table 2 summarizes the change in symptoms (LCS) and QOL (FACT-L or TOI) scores at Weeks 2, 4, and 12 from baseline following EGFR-TKI therapy. 45.7\% of patients achieved clinically meaningful improvement in symptoms (LCS $\geq 2$ points change from baseline) at Week 2 and this proportion was sustained to Week 4 (43.6\%) and Week 12 (44.6\%). The average LCS score was observed to slightly increase over baseline measurements by $1.7 \pm 0.28$ at Week 2, $2.0 \pm 0.33$ at Week 4 , and $2.0 \pm 0.34$ at Week 12 (all $\mathrm{p}<0.001)$. The average change in mean LCS at Weeks 4 and 12 were clinically meaningful.

In regards to improvement to QOL, the mean FACT-L score was observed to increase by $4.0 \pm 0.93$ at Week 2, $5.1 \pm 1.12$ at Week 4 , and $4.2 \pm 1.28$ at Week 12 (all $\mathrm{p}<0.001)$. Similarly, an increase in mean TOI score of $2.3 \pm 0.70(\mathrm{p}<0.001), 3.2 \pm 0.82(\mathrm{p}<0.001)$, and $2.4 \pm$ $0.91(\mathrm{p}=0.009)$ were observed at Weeks 2 , 4, and 12, respectively. In contrast to the meaningful change observed in LCS score, changes in mean FACT-L and TOI score were mostly not clinically meaningful.

Patients were sorted by gender, smoking status, PS, number of metastatic sites and EGFR-TKI therapy for subgroup analyses. Clinically meaningful improvement of symptoms is presented in Figure 1. Patients who were ex-smokers or with at least 3 metastatic sites were associated with increased symptoms improvement in terms of LCS. In addition, patients who are ex-smokers, with at least 3 metastatic sites, a PS of 1 or treated with gefitinib were generally associated with improved QOL in terms of TOI and FACT-L (Figure 2).

\section{Discussion}

In this prospective observational study, we provided a comprehensive assessment of symptom burden and QOL in patients with advanced NSCLC based on diverse patient-reported outcomes evaluated with FACT-L, LCS, and TOI in Taiwan. Our study indicated that patients with advanced EGFR-mutated NSCLC displayed an improvement in symptoms and QOL after the introduction of EGFR-TKI therapy. Although the overall increments 
of TOI and FACT-L did not reach defined clinically meaningful improvement (change in TOI or FACT-L $\geq$ 6 points from baseline score), the average change in LCS at week 4 and week 12 were clinically meaningful ( $\geq 2$ points change from baseline). In addition, all changes in TOI, FACT-L, and LCS of EFS population were statistically significant $(p<0.05)$. In general, the result may provide insights for clinical care.

EGFR-TKI therapy was found to be associated with a higher response rate in patients with advanced
EGFR-mutated NSCLC.[3-5] Pooled data from the LUX-Lung 3 and LUX-Lung 6 trials demonstrated a survival benefit in patients with exon 19 deletion EGFR mutations.[3] However, previous randomized trials with gefitinib and erlotinib have not shown that these patients have an increased survival advantage.[4, 5] Nevertheless, it has been demonstrated that first-line EGFR-TKIs offer an advantage compared with chemotherapy in symptom control and QOL improvements for patients with a

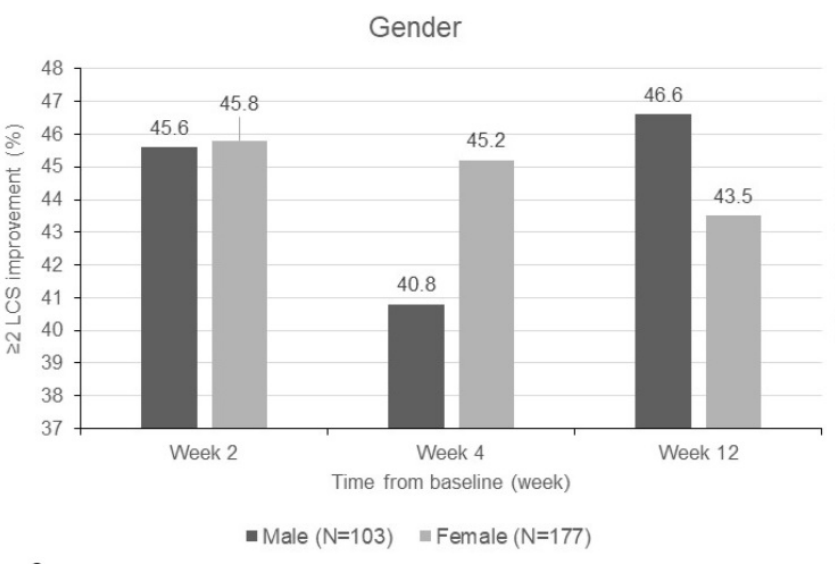

C

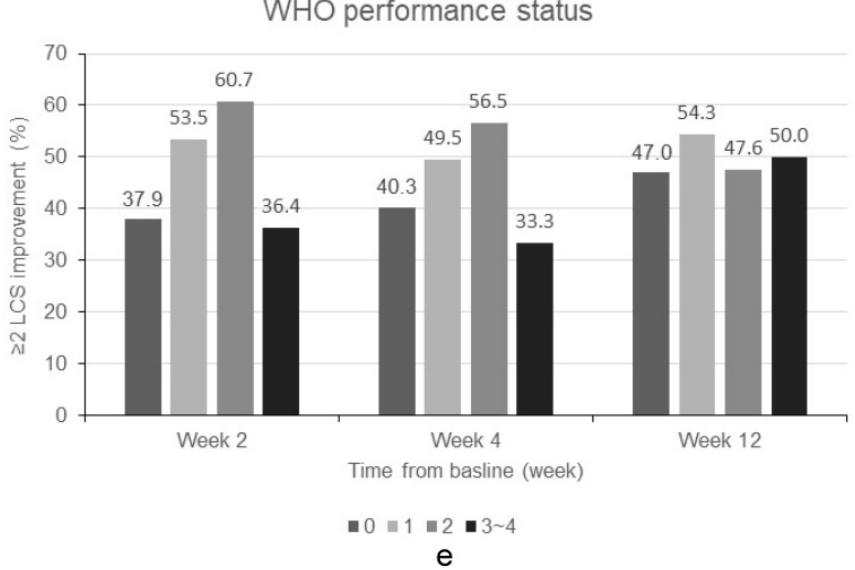

b

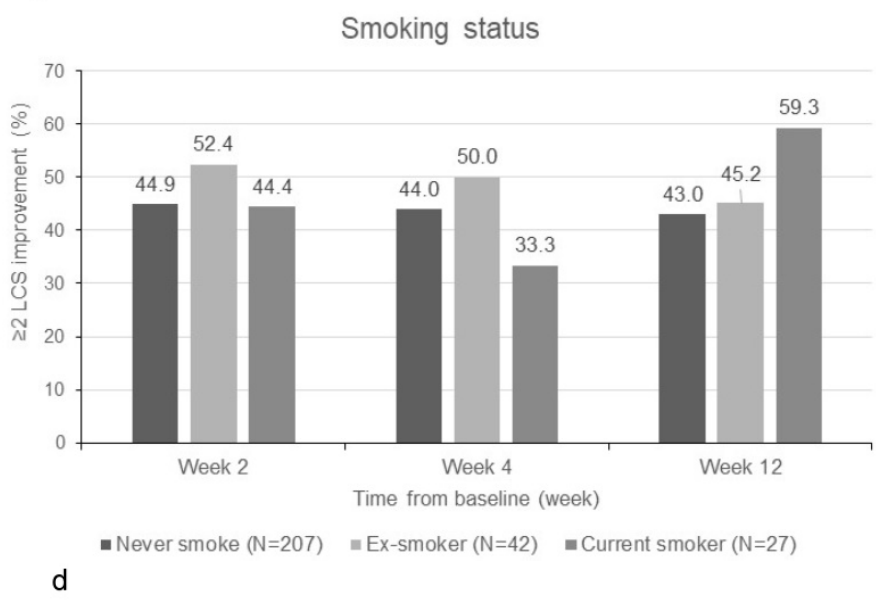

Number of metastatic sites

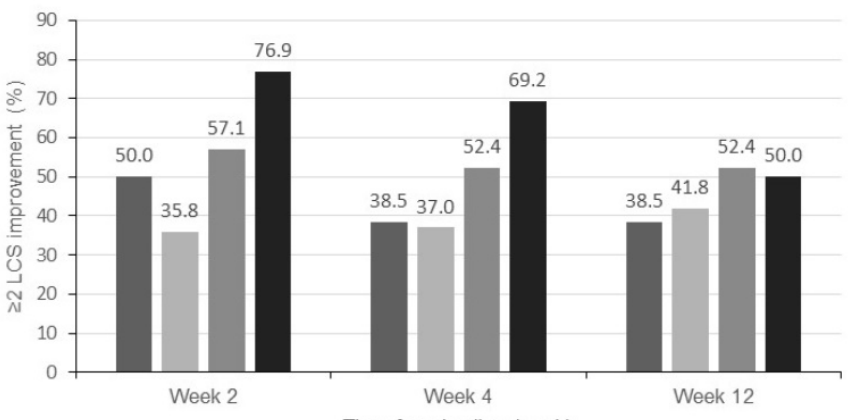

Time from basline (week)

- 0 site $(\mathrm{N}=26)=1$ site $(\mathrm{N}=165) \quad \square$ sites $(\mathrm{N}=63) \quad \square \geq 3$ sites $(\mathrm{N}=26)$

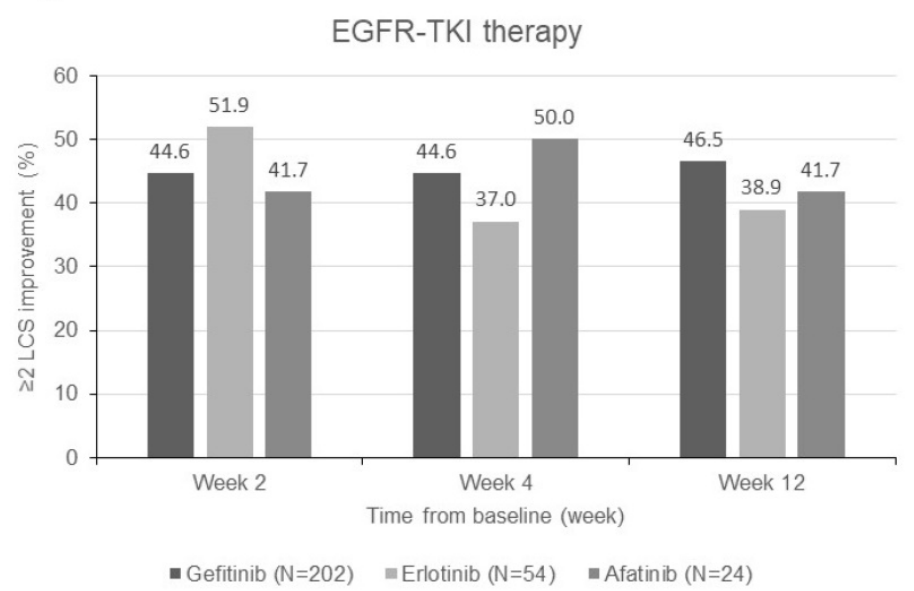

Figure 1. Subgroup analyses for clinically meaningful improvement (LCS $\geqq 2$ points from baseline score). a) gender; b) smoking status; $\mathbf{c}$ ) WHO performance status, d) number of metastatic sites and e) EGFR-TKI therapy. LCS = Lung cancer subscale; EGFR-TKI = epidermal growth factor receptor-tyrosine kinase inhibitor 
advanced EGFR-mutated NSCLC.[6-9]

In addition to patient-reported outcome assessments, the present study also provides an insight into the association of different patient characteristics with improvements in symptoms and QOL. For subgroup analyses, patients were sorted by gender, smoking status, PS, number of metastatic sites or EGFR-TKI therapy. Previous studies have shown brain and bone metastases are associated with a generally poor survival outcome and low QOL.[13, 14] Oh et al. reported tumor burden and the number of metastatic sites are predictors of poor outcomes in patients with NSCLC.[15]

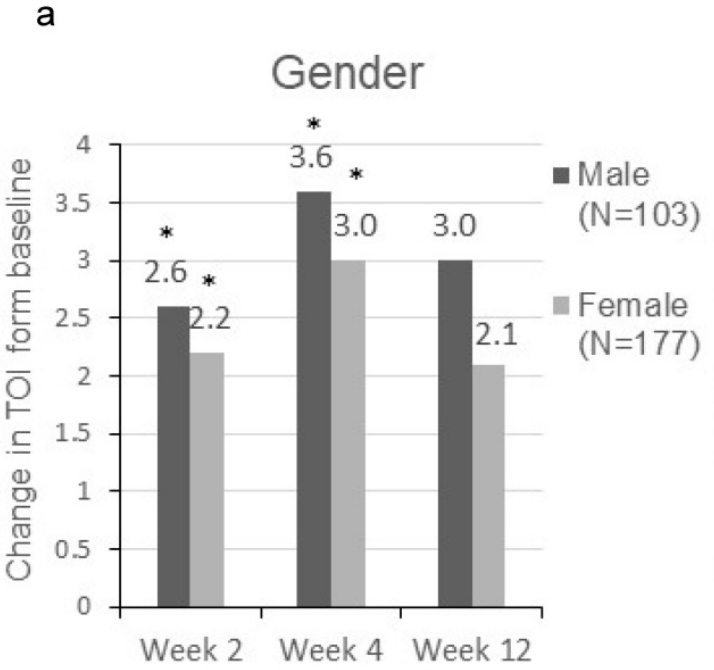

C
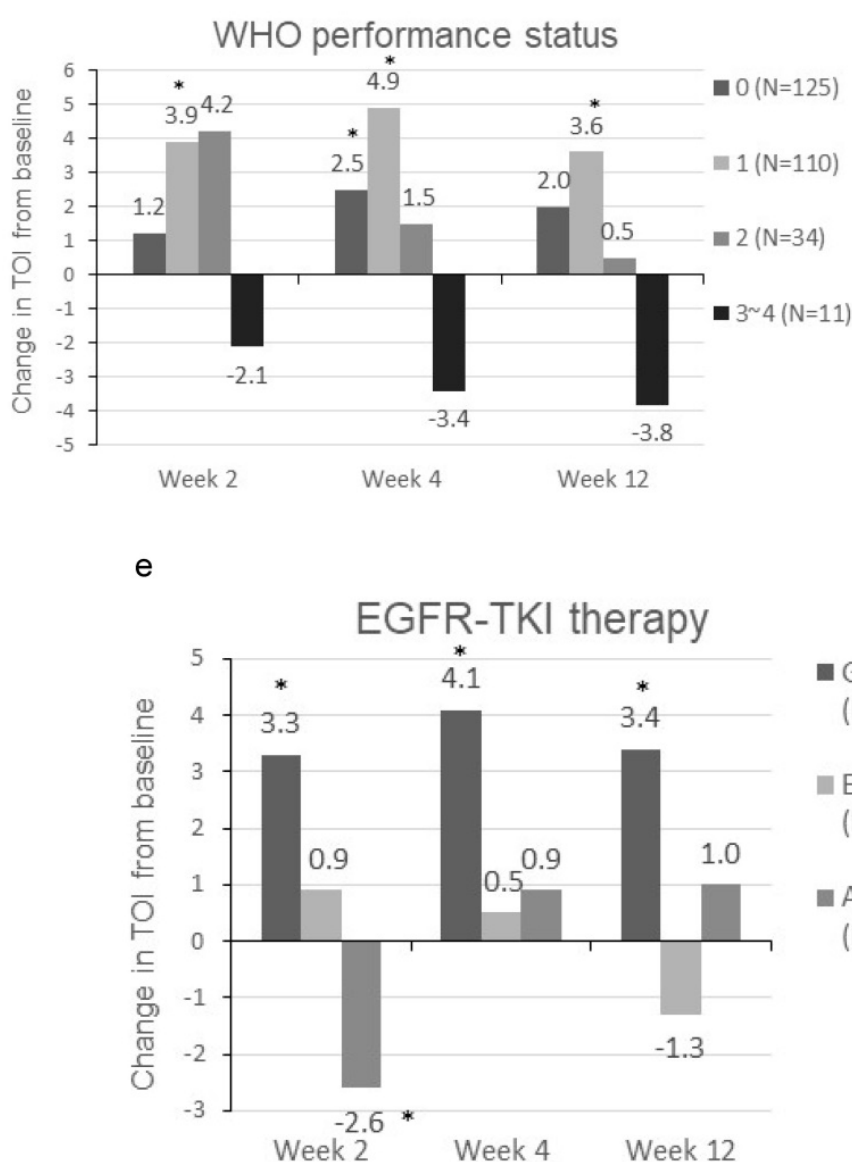

b

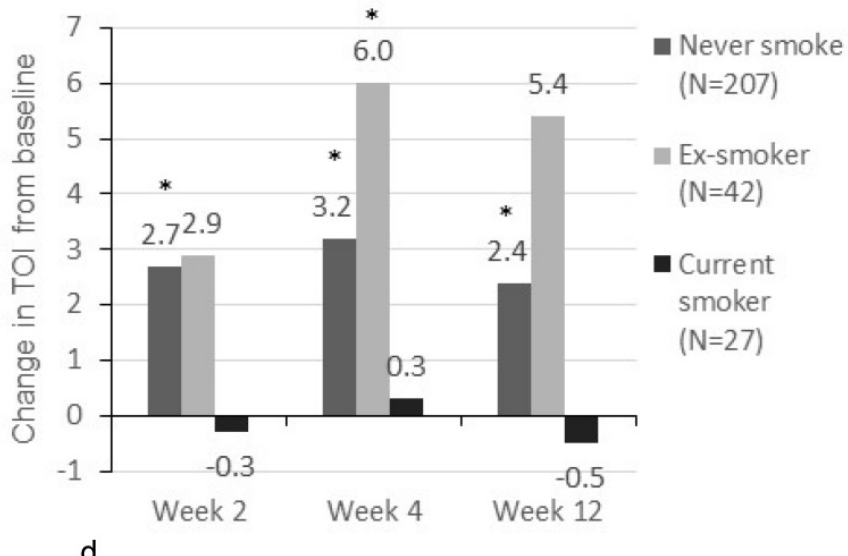

Number of metastatic sites

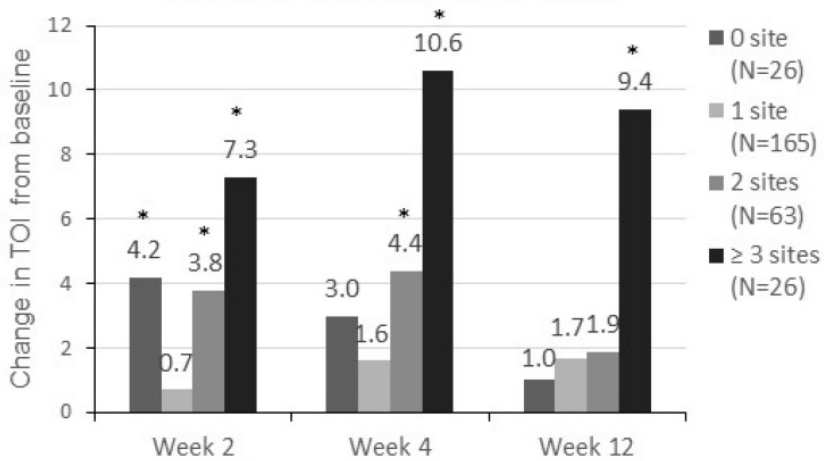

f

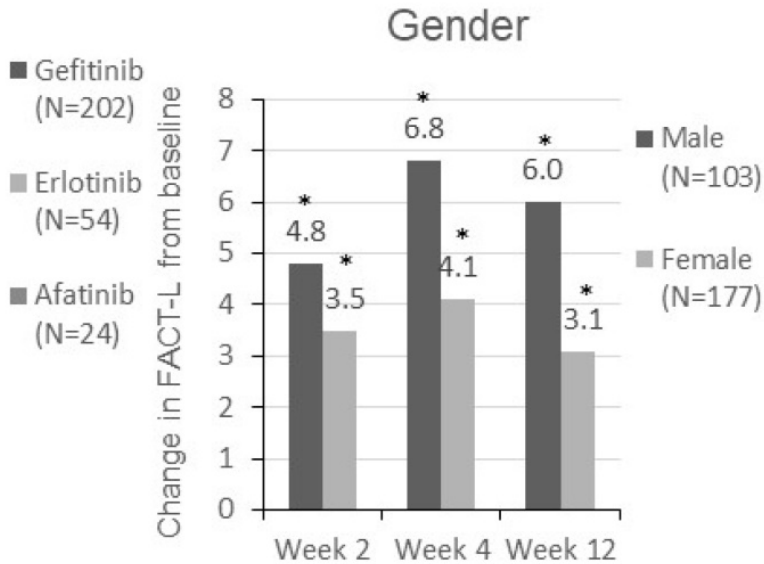


g

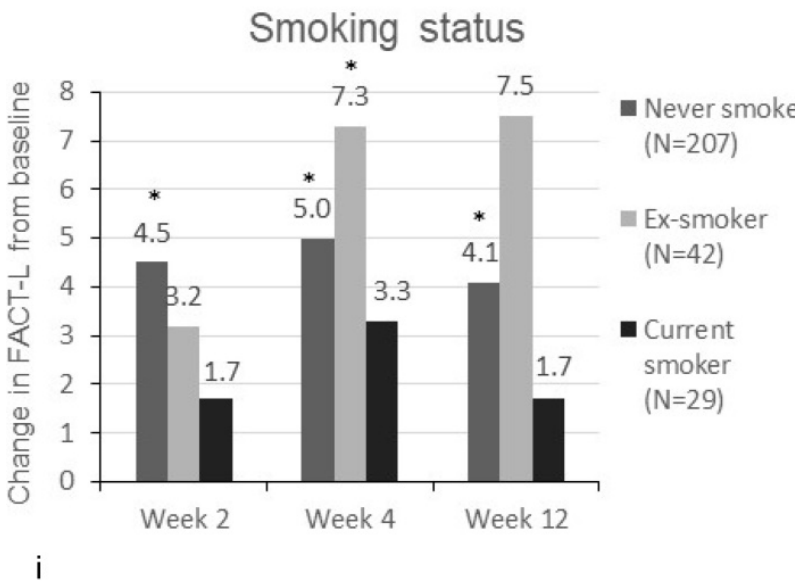

Number of metastatic sites

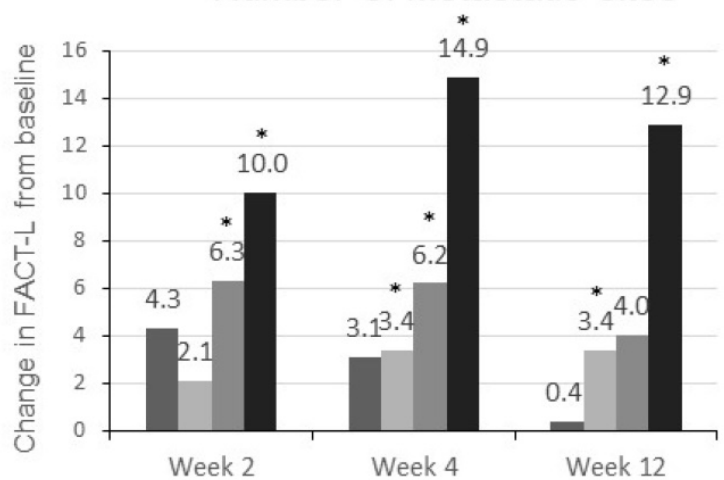

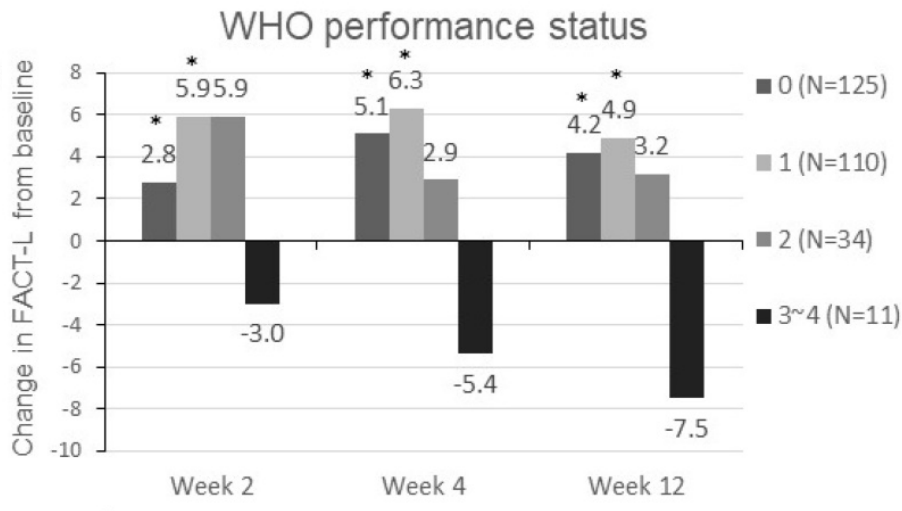

j
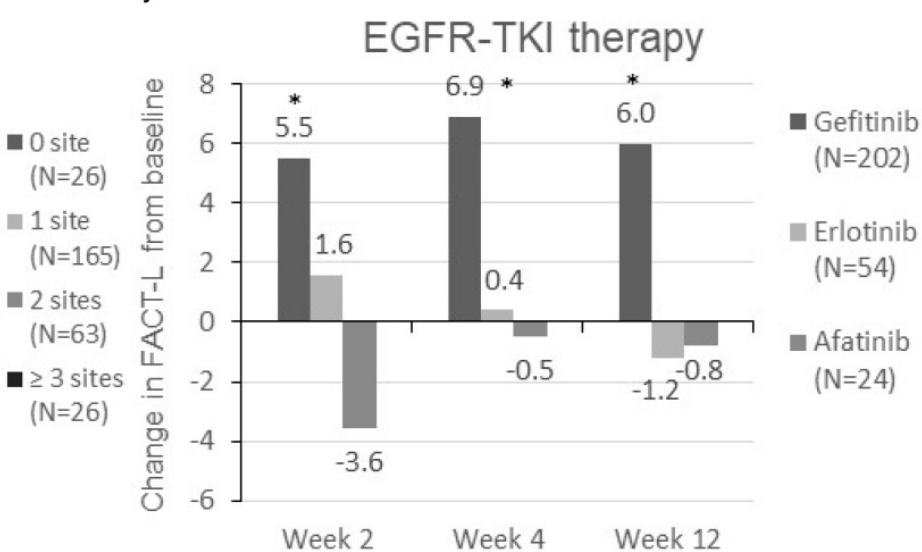

Figure 2. Change in TOI (a-e) and FACT-L (f-j) from baseline by subgroups. FACT-L = Functional Assessment of Cancer Therapy-Lung questionnaire; TOI = Treatment Outcome Index. * Score changes from baseline with $\mathrm{p}$-value $<0.05$

Smoking is another important factor associated with the worsening of symptoms and poorer QOL on diagnosis and after treatment.[16, 17] EGFR-TKIs have been demonstrated to have activity in EGFR-mutated patients with brain metastasis with a response rate of $70-80 \%$.[18] In addition, EGFR signaling is an important mediator of bone metastasis in many cancers. EGFR-TKIs may block osteoclast activation and enhance osteoblastic reactions in those patients.[19, 20] Our study indicated patients that were ex-smokers or patients with at least 3 metastatic sites, probably had worse symptoms and lower QOL at baseline and were associated with increased symptoms and QOL improvement after the introduction of EGFR-TKI treatment.

Our study also showed that patients with a PS of 1 and patients that received gefitinib treatment were also associated with improved QOL. Previous studies have indicated gefitinib and erlotinib may provide clinical benefits to EGFR-mutated patients with poor PS (s2).[21-24] Inoue and colleagues has previously reported the outcome of gefitinib treatment in 30 NSCLC patients with mutated EGFR and poor PS ( $\leq 2)$; including 22 patients with a PS of 3-4. The overall response rate to first-line gefitinib treatment was $66 \%$ and the disease control rate was $90 \%$. The rate of PS improvement was $79 \%$ and $68 \%$ of the 22 patients improved from a PS of 3-4 at baseline to a PS of 0-1 at the conclusion of the study.[23] However, a good PS at diagnosis is associated with better clinical outcome in those patients.[24, 25]

Diarrhea and skin rashes are the most frequent adverse effects related with EGFR-TKI toxicity. These adverse symptoms are observed to occur in more than half of NSCLC patients with EGFR mutation(s).[26, 27] A meta-analysis reported gefitinib was associated with lower treatment-related diarrhea and skin rash.[26] Lower treatment-related toxicities could substantially improve QOL, which was consistent with our finding in this study.

Osimertinib is a third-generation EGFR-TKI which has been approved and may now be used as a first-line treatment for advanced EGFR-mutated NSCLC patients. Treatment with osimertinib in the FLAURA trial showed significantly improved progression-free survival compared to patients on Gefitinib or Erlotinib, with a lower rate of serious adverse events.[28] However, Osimertinib may not available or affordable in certain countries. Gefitinib probably a preferred option as first-line treatment in 
those patients from the perspective of symptoms and QOL improvements.

A limitation of our study was subject to a real-world, population-based setting, the imbalanced population sorted according to different patient characteristics was inevitable. For example, most of the patients included in the study were treated with gefitinib, which was the first approved and launched EGFR-TKI in Taiwan. Relatively few patients were treated with erlotinib or afatinib in this study. In addition, the sample size was relatively small which may induce a cases bias and limit the possibility for general implications. A large-scale study is suggested for future research to collect more real-world data to confirm the trends observed in the present study.

\section{Conclusion}

Our findings suggest that symptom burden and QOL were slightly improved in EGFR-mutated advanced NSCLC patients treated with EGFR-TKIs as first-line therapy. Subgroup analyses showed that patients that were ex-smokers or with 3 or more metastatic sites were associated with improvements in symptoms and QOL. Moreover, patients with a PS of 1 or treated with gefitinib were also associated with improvement in QOL. These results may provide insights for clinical care in patients treated with EGFR-TKIs.

\section{Acknowledgements}

This study was sponsored by AstraZeneca, Taiwan. We thank the patients and their families, as well as Dr. Yu-Jen Cheng and all investigators involved in this trial.

Trial registration: ClinicalTrials.gov, NCT01947868.

\section{Competing Interests}

The authors have declared that no competing interest exists.

\section{References}

1. Torre LA, Bray F, Siegel RL, Ferlay J, Lortet-Tieulent J, Jemal A. Global cancer statistics, 2012. CA Cancer J Clin. 2015; 65: 87-108.

2. Riessk J. Shifting paradigms in non-small cell lung cancer: an evolving therapeutic landscape. Am J Manag Care. 2013; 19: s390-7.

3. Yang JC, Wu YL, Schuler M, Sebastian M, Popat S, Yamamoto N, et al. Afatinib versus cisplatin-based chemotherapy for EGFR mutation-positive lung adenocarcinoma (LUX-Lung 3 and LUX-Lung 6): analysis of overall survival data from two randomised, phase 3 trials. Lancet Oncol. 2015; 16: 141-51.

4. Mok TS, Wu YL, Thongprasert S, Yang CH, Chu DT, Saijo N, et al. Gefitinib or carboplatin-paclitaxel in pulmonary adenocarcinoma. N Engl J Med. 2009; 361: 947-57.

5. Rosell R, Carcereny E, Gervais R, Vergnenegre A, Massuti B, Felip E, et al. Erlotinib versus standard chemotherapy as first-line treatment for European patients with advanced EGFR mutation-positive non-small-cell lung cancer (EURTAC): a multicentre, open-label, randomised phase 3 trial. Lancet Oncol. 2012; 13: 239-46.
6. Geater SL, Xu CR, Zhou C, Hu CP, Feng J, Lu S, et al. Symptom and Quality of Life Improvement in LUX-Lung 6: An Open-Label Phase III Study of Afatinib Versus Cisplatin/Gemcitabine in Asian Patients With EGFR Mutation-Positive Advanced Non-small-cell Lung Cancer. J Thorac Oncol. 2015; 10: 883-9.

7. Yang JC, Hirsh V, Schuler M, Yamamoto N, O'Byrne KJ, Mok TS, et al. Symptom control and quality of life in LUX-Lung 3: a phase III study of afatinib or cisplatin/pemetrexed in patients with advanced lung adenocarcinoma with EGFR mutations. J Clin Oncol. 2013; 31: 3342-50.

8. Chen G, Feng J, Zhou C, Wu YL, Liu XQ, Wang C, et al. Quality of life (QoL) analyses from OPTIMAL (CTONG-0802), a phase III, randomised, open-label study of first-line erlotinib versus chemotherapy in patients with advanced EGFR mutation-positive non-small-cell lung cancer (NSCLC). Annals of oncology : official journal of the European Society for Medical Oncology / ESMO. 2013; 24: 1615-22.

9. Thongprasert S, Duffield E, Saijo N, Wu YL, Yang JC, Chu DT, et al. Health-related quality-of-life in a randomized phase III first-line study of gefitinib versus carboplatin/paclitaxel in clinically selected patients from Asia with advanced NSCLC (IPASS). J Thorac Oncol. 2011; 6: 1872-80.

10. Park K, Tan EH, O'Byrne K, Zhang L, Boyer M, Mok T, et al. Afatinib versus gefitinib as first-line treatment of patients with EGFR mutation-positive non-small-cell lung cancer (LUX-Lung 7): a phase 2B, open-label, randomised controlled trial. Lancet Oncol. 2016; 17: 577-89.

11. Wan C, Zhang C, Cai L, Tu X, Feng C, Luo J, et al. Psychometric properties of the Chinese version of the FACT-L for measuring quality of life in patients with lung cancer. Lung Cancer. 2007; 56: 415-21.

12. Cella D. The Functional Assessment of Cancer Therapy-Lung and Lung Cancer Subscale assess quality of life and meaningful symptom improvement in lung cancer. Seminars in oncology. 2004; 31: 11-5.

13. Peters S, Bexelius C, Munk V, Leighl N. The impact of brain metastasis on quality of life, resource utilization and survival in patients with non-small-cell lung cancer. Cancer treatment reviews. 2016; 45: 139-62.

14. Hendriks LE, Hermans BC, van den Beuken-van Everdingen $\mathrm{MH}$, Hochstenbag MM, Dingemans AM. Effect of Bisphosphonates, Denosumab, and Radioisotopes on Bone Pain and Quality of Life in Patients with Non-Small Cell Lung Cancer and Bone Metastases: A Systematic Review. J Thorac Oncol. 2016; 11: 155-73.

15. Oh Y, Taylor S, Bekele BN, Debnam JM, Allen PK, Suki D, et al. Number of metastatic sites is a strong predictor of survival in patients with nonsmall cell lung cancer with or without brain metastases. Cancer. 2009; 115: 2930-8.

16. Garces YI, Yang P, Parkinson J, Zhao X, Wampfler JA, Ebbert JO, et al. The relationship between cigarette smoking and quality of life after lung cancer diagnosis. Chest. 2004; 126: 1733-41.

17. Danson SJ, Rowland C, Rowe R, Ellis S, Crabtree C, Horsman JM, et al. The relationship between smoking and quality of life in advanced lung cancer patients: a prospective longitudinal study. Support Care Cancer. 2016; 24: 1507-16

18. Magnuson WJ, Lester-Coll NH, Wu AJ, Yang TJ, Lockney NA, Gerber NK, et al. Management of Brain Metastases in Tyrosine Kinase Inhibitor-Naive Epidermal Growth Factor Receptor-Mutant Non-Small-Cell Lung Cancer: A Retrospective Multi-Institutional Analysis. J Clin Oncol. 2017; 35: 1070-7.

19. Lu X, Wang Q, Hu G, Van Poznak C, Fleisher M, Reiss M, et al. ADAMTS1 and MMP1 proteolytically engage EGF-like ligands in an osteolytic signaling cascade for bone metastasis. Genes Dev. 2009; 23: 1882-94.

20. Pluquet E, Cadranel J, Legendre A, Faller MB, Souquet PJ, Zalcman G, et al. Osteoblastic reaction in non-small cell lung carcinoma and its association to epidermal growth factor receptor tyrosine kinase inhibitors response and prolonged survival. J Thorac Oncol. 2010; 5: 491-6.

21. Lilenbaum R, Axelrod R, Thomas S, Dowlati A, Seigel L, Albert D, et al. Randomized phase II trial of erlotinib or standard chemotherapy in patients with advanced non-small-cell lung cancer and a performance status of 2. J Clin Oncol. 2008; 26: 863-9.

22. Goss G, Ferry D, Wierzbicki R, Laurie SA, Thompson J, Biesma B, et al. Randomized phase II study of gefitinib compared with placebo in chemotherapy-naive patients with advanced non-small-cell lung cancer and poor performance status. J Clin Oncol. 2009; 27: 2253-60.

23. Inoue A, Kobayashi K, Usui K, Maemondo M, Okinaga S, Mikami I, et al. First-line gefitinib for patients with advanced non-small-cell lung cancer harboring epidermal growth factor receptor mutations without indication for chemotherapy. J Clin Oncol. 2009; 27: 1394-400.

24. Su WP, Yang CH, Yu CJ, Shih JY, Hsu C, Yang PC. Gefitinib treatment for non-small cell lung cancer -- a study including patients with poor performance status. J Formos Med Assoc. 2005; 104: 557-62. 
25. Takeda M, Okamoto I, Nakagawa K. Survival outcome assessed according to tumor response and shrinkage pattern in patients with EGFR mutation-positive non-small-cell lung cancer treated with gefitinib or erlotinib. J Thorac Oncol. 2014; 9: 200-4.

26. Ding PN, Lord SJ, Gebski V, Links M, Bray V, Gralla RJ, et al. Risk of Treatment-Related Toxicities from EGFR Tyrosine Kinase Inhibitors: A Meta-analysis of Clinical Trials of Gefitinib, Erlotinib, and Afatinib in Advanced EGFR-Mutated Non-Small Cell Lung Cancer. J Thorac Oncol. 2017; 12: 633-43.

27. Burotto M, Manasanch EE, Wilkerson J, Fojo T. Gefitinib and erlotinib in metastatic non-small cell lung cancer: a meta-analysis of toxicity and efficacy of randomized clinical trials. The oncologist. 2015; 20: 400-10.

28. Soria JC, Ohe Y, Vansteenkiste J, Reungwetwattana T, Chewaskulyong $\mathrm{B}$, Lee $\mathrm{KH}$, et al. Osimertinib in Untreated EGFR-Mutated Advanced Non-Small-Cell Lung Cancer. N Engl J Med. 2018; 378: 113-25. 